\title{
X-ray Grating Interferometry at ESRF: Applications and Recent Technical Developments
}

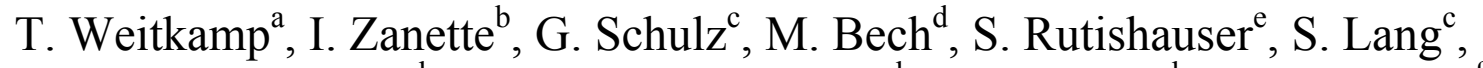

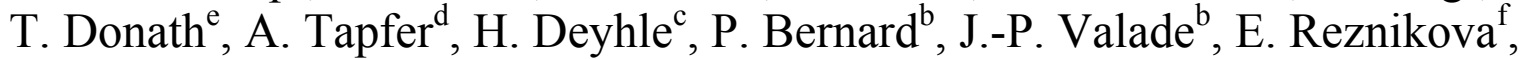 \\ J. Kenntner ${ }^{f}$, J. Mohr ${ }^{f}$, B. Müller ${ }^{c}$, F. Pfeiffer ${ }^{d}$, C. David ${ }^{\mathrm{e}}$, and J. Baruchel ${ }^{\mathrm{b}}$ \\ ${ }^{a}$ Synchrotron Soleil, Gif-sur-Yvette, France \\ ${ }^{b}$ European Synchrotron Radiation Facility (ESRF), Grenoble, France \\ ${ }^{c}$ Biomaterials Science Center, University of Basel, Basel, Switzerland \\ ${ }^{d}$ Physik-Department E17, Technische Universität München, München, Germany \\ ${ }^{e}$ Labor für Mikro- und Nanotechnologie, Paul Scherrer Institut (PSI), Villigen, Switzerland \\ ${ }^{f}$ Institut für Mikrostrukturtechnik, Karlsruher Institut für Technologie (KIT), Karlsruhe, Germany
}

\begin{abstract}
We report on the implementation of X-ray grating interferometry at the imaging beamline ID19 of the European Synchrotron Radiation Facility (ESRF). We give a brief overview of the results obtained so far with this instrument and on ongoing developments.
\end{abstract}

Keywords: Synchrotron radiation instrumentation, X-ray imaging, microtomography, X-ray phase contrast, X-ray optics PACS: 07.85.Q, 07.85.F, 41.50, 87.59.F

\section{INTRODUCTION}

X-ray grating (or Talbot) interferometry is an emerging radiography method [1-4]. It is multimodal, giving differential phase-contrast images of ultrahigh sensitivity as well as scattering contrast ("dark-field" images) [5] and absorption contrast. The method can not only be used in X-ray imaging and tomography, but also for wavefront sensing $[3,6]$ and coherence characterization applications [7, 8]. With suitable extensions, it can also be adapted to low-brilliance sources $[9,10]$.

An X-ray grating interferometer usually consists of two transmission gratings G1 and G2 (left panel in Fig. 1). The first grating G1 induces a periodic modulation on the wavefront. Ideally, the lines of G1 show only negligible absorption and shift the X-ray phase by $\pi / 2$ or $\pi$. Downstream of G1, the phase modulation will transform into an intensity modulation. For an incident plane wave, maximum intensity contrast is observed at distances $D_{n}=$ $n p_{1}{ }^{2} /\left(2 \lambda \eta^{2}\right)$ from G1, where $n$ is any odd integer number, $p_{1}$ the period of $\mathrm{G} 1, \lambda$ the X-ray wavelength, $\eta=1$ for a $\pi / 2$ shifting grating, and $\eta=2$ for a $\pi$-shifting grating [11]. The intensity modulation period will be $p_{1} / \eta$.

A second grating $\mathrm{G} 2$ with period $p_{1}=p_{1} / \eta$ and strongly absorbing structures is placed in one of the planes of maximum contrast. It acts as a transmission mask for the detector and allows determination of modulation fringe position and amplitude, even if the fringe spacing is much smaller than the pixel size of the detector [2-4].

A grating interferometer of this type can be used in moiré [12] or phase-stepping mode [4, 13]. Moiré mode gives all information in one shot, at the cost of spatial resolution. Phase stepping makes full use of the spatial resolution of the imaging system, but requires that one of the two grids be scanned over one period in three or more steps.

When combined with X-ray tomography, the differential phase contrast capacity of grating interferometry yields quantitative reconstructions of the three-dimensional distribution $\delta(x, y, z)$ of the decrement of X-ray refractive index. The sensitivity of grating interferometric phase tomography has been proved to be as good as $2 \times 10^{-10}$ (expressed in terms of the standard deviation of reconstructed values of $\delta$ in a homogeneous region of the sample) [14-16].

The 10th International Conference on X-ray Microscopy

AIP Conf. Proc. 1365, 28-31 (2011); doi: 10.1063/1.3625297

(C) 2011 American Institute of Physics 978-0-7354-0925-5/\$30.00 


\section{GRATING INTERFEROMETRY AT ID19}

The 150-m-long beamline ID19 at the ESRF is fully dedicated to full-field parallel-beam imaging, with a focus on tomography $[17,18]$. Located on a low- $\beta$ section of the storage ring, it delivers $\mathrm{X}$ rays with energies ranging from 7 to more than $100 \mathrm{keV}$, supplied by four insertion devices. In its present configuration $[18,19]$, the maximum beam size is $40 \mathrm{~mm}$ (width) by $15 \mathrm{~mm}$ (height). In the development of X-ray grating interferometry in Europe, ID19 has played an important part and continues to do so [14, 16, 20,21].
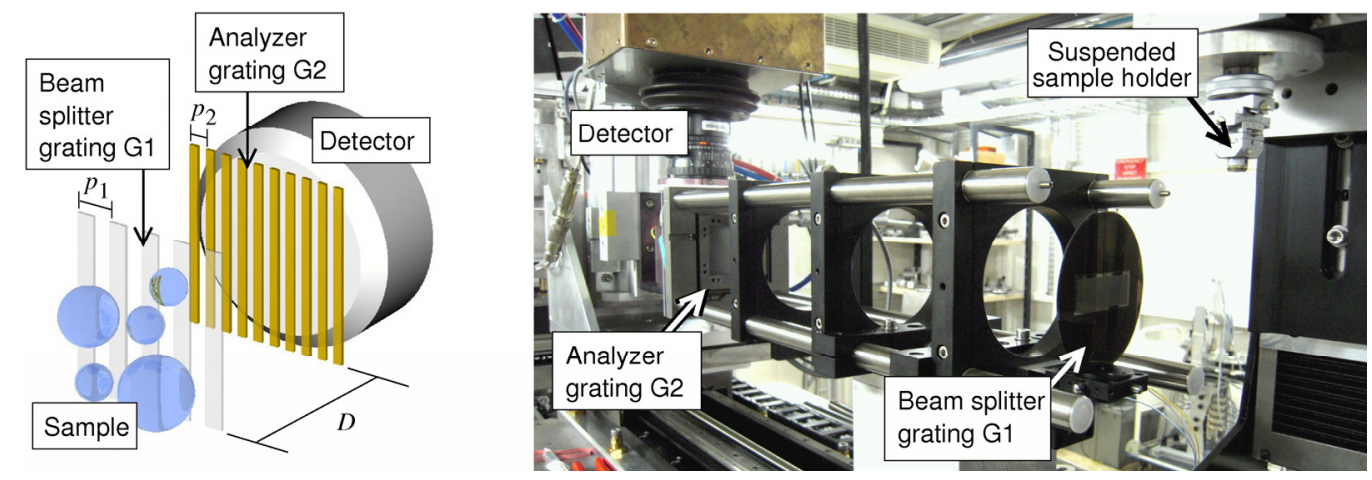

FIGURE 1. Left: Setup of a grating interferometer for X-ray imaging (schematic). Right: Photograph of the large-field interferometer setup on ID19.

A dedicated interferometer setup has been installed at the beamline since the year 2008. Today, two modular interferometer hardware systems are available at ID19. Each of these two systems is used as a removable add-on to one of the two permanently installed tomography setups of the beamline. Both interferometer systems are based on standard commercial optical mount structures. This concept, originally developed at the Paul Scherrer Institut, has proved flexible and stable. The gratings used are fabricated by collaboration partners (PSI, KIT) using specifically developed microstructuring processes [22-24].

The more commonly used of the two systems gives access to the full beam size at ID19. It is optimized for high sensitivity and large field of view. Mounted on the ID19 medium-resolution tomograph [18], this system is typically used with the crystal monochromator of the beamline. Figure 1 (right panel) shows a picture of the device. Technical details can be found elsewhere [25]. Beam splitter gratings for this interferometer are available for X-ray photon energies of 17.6, 23, and $35 \mathrm{keV}$. An experimental beam splitter with a design energy of $82 \mathrm{keV}$ was also tested.

The large-field interferometer is generally used with a purpose-made tomography sample stage in which the sample is suspended so it can easily be immersed in a water tank during measurement, to avoid artifacts from the very strong phase gradient at the surface or the container wall.

A second interferometer system for smaller fields, but higher spatial resolution, is currently used for in-house studies. This system, too, is based on a concept developed at the Paul Scherrer Institut and adapted to the ESRF environment [25]. It can be used with the ID19 multilayer monochromator.

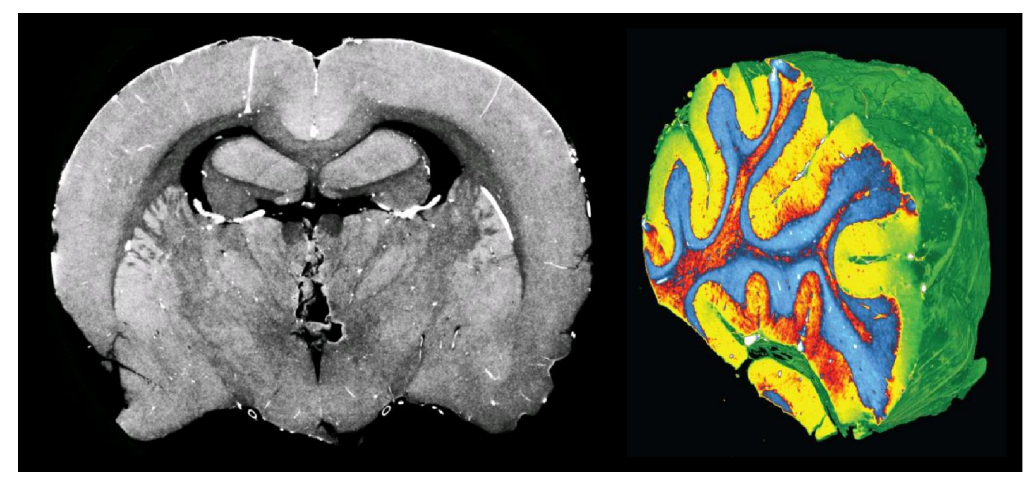

FIGURE 2. Left: Phase tomogram of a rat brain in buffered phosphate solution [26], obtained with the large-field interferometer. Right: Three-dimensional false-color rendering of a human cerebellum, from a phase tomography dataset obtained with the interferometer. The full study is reported elsewhere [16]. 
The data processing chain is based on the phase stepping mode and essentially comprises two steps. In the first step, the phase-stepping scan data are analyzed using computer code written by beamline staff. This code converts the original raw data into pseudo-absorption, differential phase contrast, and dark-field images for each tomography projection angle. In the second step, the tomographic reconstruction is performed with the standard ESRF tomography reconstruction program PyHST, using the processed projection images generated in the previous step. A Hilbert filter for processing of DPC data [27] is implemented in PyHST. Figure 2 shows examples of phase tomography results obtained with this processing chain.

\section{TWO-DIMENSIONAL INTERFEROMETER}

To complement the information obtained with a grating interferometer, the concept can be extended by using two-dimensional (2D) structures as gratings G1 and G2 (Fig. 3), rather than the line gratings commonly used. Such a 2D interferometer gives contrast in both transverse directions and can thus yield quantitative projection phase maps of better quality than a one-dimensional device (i.e., using line gratings). In addition, a 2D interferometer gives access to information on the orientation of scattering structures in the specimen ("directional dark-field imaging").

Numerical studies show that a suitable combination of 2D grating structures can be obtained by superimposing two sets of one-dimensional gratings and that the quality of the phase projection images obtained can be expected to be far superior to those from a conventional one-dimensional device [28, 29]. These predictions could be verified in a recent experiment on ID19 [30]. A 2D interferometer can be especially useful when projection radiography without tomographic reconstruction is used, for fast imaging (including fast tomography) in moiré mode [31], and for wavefront sensing at highly brilliant synchrotron or X-ray free-electron laser (XFEL) sources.

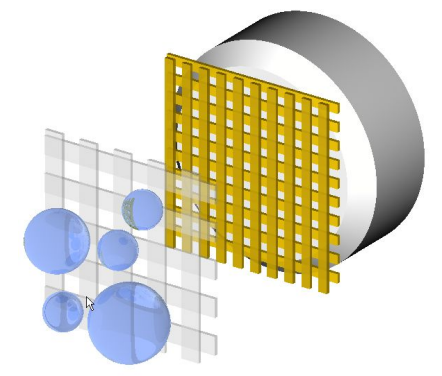

FIGURE 3. Two-dimensional grating interferometer (schematic). See also dedicated article by Zanette et al. [28] in this volume.

\section{CONCLUSIONS AND OUTLOOK}

In collaboration with several laboratories throughout Europe, an X-ray grating interferometer setup was developed and installed at beamline ID19 of the European Synchrotron Radiation Facility in Grenoble, France. Its modular nature gives access to a wide range of energies and Talbot distances. With this device, ID19 now provides a highly sensitive and reliable instrument for ultra-sensitive grating-based X-ray phase tomography. The high spatial coherence, large beam, and wide energy range of ID19 make this instrument a unique tool for the life sciences, paleontology, and materials science.

Among the ongoing developments are the access to higher X-ray energies and the development of twodimensional interferometry. Access to high energies is crucial for the further development of synchrotron-based applications in paleontology, i.e., the imaging of fossilized organisms, and as a test bed in view of the potential future use of interferometry outside a synchrotron environment, for example in clinical imaging (mammography, head or body CT). The use of 2D gratings, rather than the current line gratings, and the development of related measurement and analysis strategies, can substantially improve data quality and phase reconstructions, particularly in cases where high accuracy of the results is a priority.

\section{ACKNOWLEDGMENTS}

The authors gratefully acknowledge all past and present staff of PSI and KIT who have contributed to the development of grating fabrication processes. Members of the ESRF groups for scientific software and beamline 
controls are acknowledged for the grating-specific additions to the PyHST tomography reconstruction software (A. Mirone) and for their assistance in implementing the interferometer into the beamline (J. M. Clement and A. Homs).

This work was carried out with the support of the Karlsruhe Nano Micro Facility (KNMF), a Helmholtz Research Infrastructure at Karlsruhe Institute of Technology (KIT). The European Synchrotron Radiation Facility (ESRF) has granted beamtime for this project through proposals MD-404, MD-407, MD-408, MI-983, MI-1058.

\section{REFERENCES}

1. C. David, B. Nöhammer, H. H. Solak, and E. Ziegler, Appl. Phys. Lett. 81, 3287 (2002).

2. A. Momose, Jpn. J. Appl. Phys. 42, L866 (2003).

3. T. Weitkamp, B. Nöhammer, A. Diaz, C. David, and E. Ziegler, Appl. Phys. Lett. 86, 054101 (2005), and Erratum [32].

4. T. Weitkamp et al., Opt. Express 13, 6296 (2005).

5. F. Pfeiffer et al., Nat. Mater. 7, 134 (2008).

6. E. Ziegler, L. Peverini, I. V. Kozhevnikov, T. Weitkamp, and C. David, AIP Conf. Proc. 879, 778 (2007).

7. F. Pfeiffer et al., Phys. Rev. Lett. 94, 164801 (2005).

8. A. Diaz et al., J. Synchrotron Radiat. 17, 299 (2010).

9. F. Pfeiffer, T. Weitkamp, O. Bunk, and C. David, Nature Physics 2, 258 (2006).

10. C. Kottler et al., Physica stat. sol. A 204, 2728 (2007).

11. T. Weitkamp, C. David, C. Kottler, O. Bunk, and F. Pfeiffer, Proc. SPIE 6318, 63180S.1 (2006).

12. T.Weitkamp et al., Proc. SPIE 5533, 140 (2004).

13. T. Weitkamp et al., SPIE 5535, 137 (2004).

14. F. Pfeiffer et al., Phys. Med. Biol. 52, 6923 (2007).

15. J. Herzen et al., Opt. Express 17, 10010 (2009).

16. G. Schulz et al., J. Roy. Soc. Interface (2010), doi:10.1098/rsif.2010.0281, in press.

17 P. Cloetens et al. J. Phys. D 32, A145 (1999).

18 T. Weitkamp et al., AIP Conf. Proc. 1221, 33 (2010).

19. T. Weitkamp et al., AIP Conf. Proc. 1234, 83 (2010).

20. T. Weitkamp et al., Eur. J. Radiol. 68, S13 (2008).

21. B. Müller et al., Proc. SPIE 7078, 70780B.1 (2008).

22. C. David et al., Microelectron. Eng. 84, 1172 (2007).

23. E. Reznikova, J. Mohr, M. Börner, V. Nazmov, and P. J. Jakobs, Microsyst. Technol. 14, 1683 (2008).

24. J. Kenntner et al., Proc. SPIE 7804, 780408 (2010).

25. T. Weitkamp et al., Proc. SPIE 7804, 780406 (2010).

26. F. Pfeiffer et al., J. Phys. Conf. Series 186, 012046 (2009).

27. F. Pfeiffer, C. Kottler, O. Bunk, and C. David, Phys. Rev. Lett. 98, 108105 (2007).

28. I. Zanette, S. Rutishauser, C. David, and T. Weitkamp, these proceedings.

29. I. Zanette, C. David, S. Rutishauser, and T. Weitkamp, AIP Conf. Proc. 1221, 73 (2010).

30. I. Zanette, T. Weitkamp, T. Donath, S. Rutishauser, and C. David, Phys. Rev. Lett. 105, 248102 (2010).

31. A. Momose, W. Yashiro, H. Maikusa, and Y. Takeda, Opt. Express 17, 12540 (2009).

32. T. Weitkamp, B. Nöhammer, A. Diaz, C. David, and E. Ziegler, Appl. Phys. Lett. 86, 119902 (2005). 